\title{
Semen Stain Detection: A Study on the Different Fabrics for Effects of Washing
}

\author{
Awasthi $\mathrm{A}^{1}$, Jain $\mathrm{B}^{1 *}$ and Mishra $\mathrm{G}^{2}$ \\ ${ }^{1}$ University of Lucknow, India \\ ${ }^{2}$ Department of Anthropolgy, University of Lucknow, India
}

*Corresponding author: Bharti Jain, University of Lucknow, Lucknow, India, Email: bhartijain871@gmail.com

\section{Research Article \\ Volume 5 Issue 4}

Received Date: October 28, 2020

Published Date: November 18, 2020

DOI: $10.23880 /$ ijfsc-16000207

\section{Abstract}

As the number of rape cases are increasing day by day. There is a requirement of detection of seminal fluid and sperm on various surfaces, even if semen and seminal fluid available in few quantities. The present work focuses to determine three possible aspects affecting semen determination on laundered clothing: the temperature of water while laundering and handling if the stain was dry or wet at the period of laundering of the fabric form. During present work, four types of fabrics (Polyester, Denim, Cotton and wool) were analysed microscopically by applying of methods to determine semen (U.V. light source, Acid phosphatase test) the outcome demonstrates the significance of the methodology and precautions to be taken whenever evaluating findings for the identification of semen, in general on objects which may have been laundered.

Keywords: Semen; Laundering; Acid Phosphatase; Microscopic Examination

\section{Introduction}

Seminal fluid is a blend of cells, sperm and a combination of inorganic and organic substances. It is very dense and gelatinous material which is produced in males by seminal vesicle, prostate and cowper's glands [1]. "Seminogram" also knows as semen analysis. Semen analysis is the determination of ejaculated seminal fluid collected from a crime scene. An average male ejaculate approximately $3.5 \mathrm{ml}$ of seminal fluid. Every milliliter can generate around 10-50 million sperm cells. These figures may differ with the age of the male and may be adversely affected by medical conditions, genetic history, diet, smoking habits and the use of illegal drugs [2].

Just a few printed experiments have been performed to detect and classify semen or spermatozoa after processing. Traces of sperm cells retrieved on clothing require challenges as to the explanation of such findings in rape cases [3-6]. The way of discharge may not be readily identified, clothing may have been washed, or the findings may not be related to the incidence under investigation [7]. A few recent researches have focused on semen collection from laundered specimens and in most of these reports cotton has been used as the most common fabric type. However, there are limited studies on the semen stain collected from different types of fabrics and the laundering conditions. The present study aims to analyze the effect of different temperatures of laundering, fabric type on semen stain which can be used as evidence in cases of sexual assault.

\section{Methods and Materials}

Samples of semen have been obtained by volunteers with informed written consent. $150 \mu \mathrm{L}$ of semen was deposited onto four types of fabrics; polyester, denim, cotton and wool. Unwashed positive controls were used, where every specimen was organised in a single sample. It was repeated in terms of developing 4 classes dependent on the laundering 
situations;

- Hot water with dry stains

- Hot water with wet stains

- Cold water with dry stains

- Cold water with wet stains

Unwashed positive controls were used, where every specimen was organised in a single sample (Table 1).

\begin{tabular}{|c|c|c|c|c|}
\hline & Polyester & Denim & Wool & Cotton \\
\hline Hot/Dry & 1 & 1 & 1 & 1 \\
\hline Hot/Wet & 1 & 1 & 1 & 1 \\
\hline Cold/Dry & 1 & 1 & 1 & 1 \\
\hline Cold/Wet & 1 & 1 & 1 & 1 \\
\hline Unwashed & 1 & 1 & 1 & 1 \\
\hline
\end{tabular}

Table 1: Planned data set.

Every sample was laundered during either the hot usual period $\left(\sim 50^{\circ} \mathrm{C}\right)$ or the cold usual period $\left(\sim 20^{\circ} \mathrm{C}\right)$. Moist spots were laundered within 30 minutes of depositing the semen. During each of the washing cycles, no detergent had been used, and specimens became dry after washing under shade.

\section{Alternate Light Source}

All the samples have been analysed with U.V. a $\mathrm{t}$ a 455 $n m$ wavelength. The smudge's fluorescence was reported as solid, moderate, weak, or undetected.

\section{Acid Phosphatase Testing}

All the samples have been analysed using the AP test process. Firstly put a tiny portion of fabric of seminal stain and then add few drops of alpha-naphthol phosphate and brentamine fast blue $B$ reagents but that has been freshly prepared. If a purple colour reaction takes place within ten sec., a positive result was noted. If there was no colour change within ten sec. the sample was considered negative.

\section{Christmas Tree Staining}

Samples collected from the fabric after the AP test in order to take out the sperm cells from its cloth piece. In the case of samples which were positive for AP, the portion was collected from the centre of the region in which the highest positive AP reactions were reported. Samples were collected using established procedures by using sterile $\mathrm{ddH}_{2} \mathrm{O}$, softening the stain, putting the fabric in a spin jar, and rotating the sample down to create a pellet. The pellet was

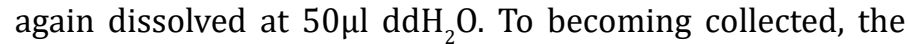
samples were poured onto a glass slide, dried and stained with Nuclear Fast Red for 15 minutes. When the primary stain was washed, the picro-indigocarmine secondary stain was added for 15 seconds and again washed. After the slides had been fully dried, the cover lips were assembled using mounting media.

Slides were examined under microscope and classified depend on the amount of spermatozoa present in Table 2.

\begin{tabular}{|c|c|}
\hline Density of Sperm & Score \\
\hline No noticeable sperm & Negative \\
\hline Hard to find sperm & $1+$ \\
\hline Numerous sperm in some areas & $2+$ \\
\hline In most areas, numerous or definite sperm & $3+$ \\
\hline Multiple sperm in every field & $4+$ \\
\hline
\end{tabular}

Table 2: Score system utilised examine slides made from the Christmas tree staining.

\section{Results}

\section{Alternate Light Source}

The unclean samples displayed a degree of uncertainty for the ALS test, depending on the form of fabric on which the spot was found (Table 3). Although the semen stain luminance heavily on the cotton, the stain on the wool displayed minimal luminescence, although any denim luminescence was very hard to spot. The polyester displayed absolutely no luminescence. Only the stains which are dry at the period of laundering, and then only continuously on the cotton, were found for washed stains. When the smear was dried and washed in cold water the wool revealed a slight degree of fluorescence. During laundry time, none of the stains that were wet displayed fluorescence.

\begin{tabular}{|c|c|c|c|c|}
\hline & Denim & Wool & Polyester & Cotton \\
\hline Unwashed & Weak & Moderate & Negative & Strong \\
\hline Cold/Dry & Negative & Weak & Negative & Weak \\
\hline Cold/Wet & Negative & Negative & Negative & Negative \\
\hline Hot/Dry & Negative & Negative & Negative & Weak \\
\hline Hot/Wet & Negative & Negative & Negative & Negative \\
\hline
\end{tabular}

Table 3: Results of ALS Examination of Samples.

\section{Acid Phosphatase Test}

All samples revealed preliminary colour formation in under ten seconds once the AP reagent had been implemented for the AP test (Figure 1). During the period of laundering none of the spots which were damp tested positive for suspected semen involvement. Even polyester produced diverse outcomes of the dry stains, with every 


\begin{tabular}{|c|c|c|c|c|}
\hline & Polyester & Wool & Denim & Cotton \\
\hline Unwashed & POSITIVE & POSITIVE & POSITIVE & POSITIVE \\
\hline Hot/Dry & POSITIVE & POSITIVE & POSITIVE & POSITIVE \\
\hline Hot/Wet & NEGATIVE & NEGATIVE & NEGATIVE & POSITIVE \\
\hline Cold/Dry & POSITIVE & POSITIVE & POSITIVE & POSITIVE \\
\hline Cold/Wet & NEGATIVE & NEGATIVE & NEGATIVE & POSITIVE \\
\hline
\end{tabular}

Table 4: Results of AP test.

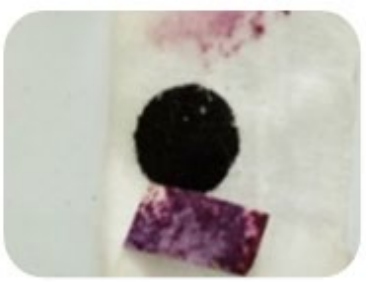

(a) Unwashed

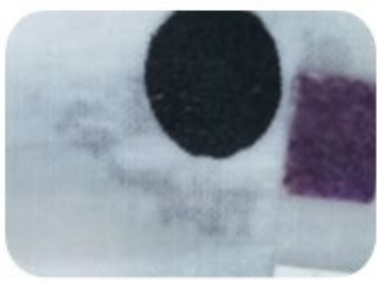

(b) Cold water wet semen stain

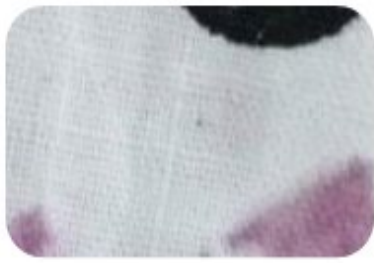

(c) Cold water dry semen stain

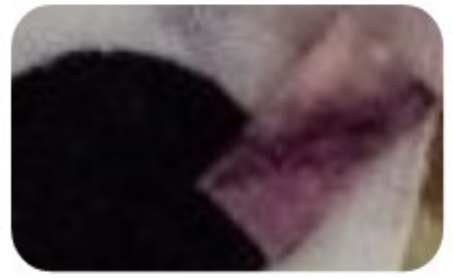

(d) Hot water wet semen stain

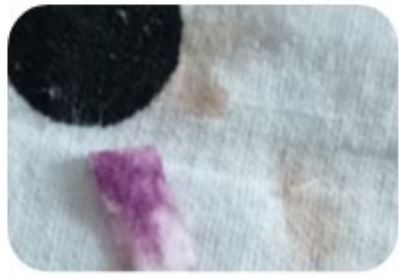

(e) Hot water dry semen stain

Figure 1: Relevant images after AP mapping of cotton fiber. a. Unwashed cotton control sample. b. The cotton sample was washed in cold while the stain was still wet. c. The cotton sample was washed in cold water after drying the stain. d. Cotton sample washed in hot water while the stain was still wet. e. The cotton sample was washed in hot water after drying the stain.

\section{Christmas Tree Staining}

Every samples positively identified for the existence of spermatozoa for the Christmas tree staining, and scores of $2+$ or $3+$ have been issued. The majority of the specimens displayed inconstant outcomes and none of the samples washed obtained a point beyond $1+$. While laundering the denim specimens retained the most spermatozoa, since there was still one denim sample which tested negative for spermatozoa. The scoring system is shown in Table5.

\begin{tabular}{|c|c|c|c|c|}
\hline & Cotton & Polyester & Denim & Wool \\
\hline Hot/Dry & +1 & +1 & +1 & +1 \\
\hline Hot/Wet & +1 & +1 & +1 & +1 \\
\hline Cold/Dry & +1 & +1 & +1 & +1 \\
\hline Cold/Wet & +1 & +1 & +1 & +1 \\
\hline Unwashed & +3 & +3 & +3 & +3 \\
\hline
\end{tabular}

Table 5: Microscopic test findings for christmas tree stains. 


\section{International Journal of Forensic Sciences}

\section{Conclusion}

The outcome of this research offers useful knowledge to all Law Enforcement officials as well as people in field of Forensic Science. It not only demonstrates but also tell us the precautionary measures used to be taken before and after semen sample collection whenever evaluating the findings from the gathered sample. Most crucial step to do research in that is as they have very short lifespan so if the packing of sample from laundry is not appropriate then we might lose everything. Many researches are going on in field of semen be it through Hydrogel technique or spectroscopy. When the sample has completed its preliminary test and samples are sent for DNA analysis then we get the final result which is further matched with culprit's sample swab who committed such heinous crime.

However, there are a few drawbacks of semen detection techniques for stains found on washed fabrics in the present study. Washing will seriously inhibit the procedure of Alternate Light Source to detect possible biological spots, at the moment it was washed away, it was due to the water temperature or whether the stain was wet or dry. The AP test may undoubtedly identify the existence of semen irrespective of the temperature of water whenever washed; but, if the stain is even moist at the period of laundry, the detection of acid phosphatase can be seriously impeded. In addition, the form of fabric could have a significant effect, as the acid phosphatase test was inadequate in spotting semen stains on polyester. Though, these differences didn't occur in any other cotton clothing used during the research. Therefore, further studies need to be done with more samples with improved and specific techniques.

\section{Acknowledgment}

I would like to thank the State Forensic Science
Laboratory at Lucknow as well as Cytogenetic Laboratory of Anthropology Department, University of Lucknow, for the use of their resources and facilities throughout the completion of this project.

\section{References}

1. Puga Molina LC, Luque GM, Balestrini PA, Marín Briggiler CI, Romarowski A, et al. (2018) Molecular Basis of Human Sperm Capacitation. Frontiers in Cell and Developmental Biology 6: 72.

2. Kumar N, Singh U (2018) Forensic Analysis of Semen : A Review. International Journal of Information And Computing Science 5(9): 81-86.

3. Viner TC, Kagan RA, Johnson JL (2014) Using an alternate light source to detect electrically singed feathers and hair in a forensic setting. Forensic Science International 234: e25-e29.

4. Spector J, Von Gemmingen D (1971) The effect of washing on the detection of blood and seminal stains. Journal of the Canadian Society of Forensic Science 4(1): 3-9.

5. Farmen RK, Cortez P, Frøyland ES (2008) Spermatozoa recovered on laundered clothing. Forensic Science International: Genetics Supplement Series 1(1): 418420.

6. Nelson DG, Santucci KA (2002) An alternate light source to detect semen. Academic Emergency Medicine 9(10): 1045-1048.

7. Kobus HJ, Silenieks E, Scharnberg J (2002) Improving the Effectiveness of Fluorescence for the Detection of Semen Stains on Fabrics. Journal of Forensic Sciences 47(4): $1-5$.

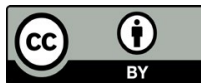

